\title{
A Health Monitoring Study of Multiple-Unit Train Braking System using Sample Identification Approach
}

\author{
Bo Wang ${ }^{1}$ Yang $\mathrm{Ji}^{1}$ Bohui Liu ${ }^{1}$ Feng Gao $^{2}$ Weijun Yang ${ }^{2}$ Dunwen Gan ${ }^{2}$ \\ ${ }^{1}$ Shenyang Yuanda Simtek Co., Ltd., China, \{bo.wang, yang.ji, bohui. liu\} @ cnydsimtek. com \\ ${ }^{2}$ Beijing Zongheng Electro-Mechanical Technology Development Co. , China, \\ \{gaofeng, yangwei jun, gandunwen\} @ zemt. cn
}

\begin{abstract}
The development of the Chinese high-speed railway has experienced considerable dynamics in recent years. Further development of the trains depends to a large extent on the optimization of the key subsystems and monitoring of reliability and safety. In this paper the sample identification based health monitoring method for fault diagnosis and health monitoring by use of a Modelica based model of the train system is addressed. In this study the proposed method sovled the issue of generating enough fault samples for health monitoring and be validated by the train operation realtime data. The paper demonstrates the applicability of the method and modeling concept by means of diagnosis and monitoring of the state of health of the braking system.
\end{abstract}

Keywords: high speed train, braking system, health monitoring

\section{Introduction}

\subsection{Development of Chinese railway and re- cent activities in high speed train develop- ment}

The Chinese high speed railway (HSR) was first introduced in 2007 designed for the speeds of $250-350 \mathrm{~km} / \mathrm{h}$. In 2008, the world's first high speed rail with a designed speed of $380 \mathrm{~km} / \mathrm{h}$ between Beijing and Shanghai was in operation. In mid-2018, the HSR has extended to 30 provincial-level administrative divisions and reached $27000 \mathrm{~km}$ in total length (author?) (1). Since 2008, China has developed CRH trains (CRH1, CRH2, CRH3 and CRH5) with a maximum velocity of $300-350 \mathrm{~km} / \mathrm{h}$. In 2010, a new generation of CRH trains with a top speed of $380 \mathrm{~km} / \mathrm{h}$ were developed and entered service on the Shanghai-Hangzhou High Speed Railway. The newest trains CR400AF and CR400BF called "Fuxing Hao" in figure $1 \mathrm{can}$ reach the top speed of $400 \mathrm{~km} / \mathrm{h}$ narrowing the commuting time from 6 hours to 4.5 hours between Beijing and Shanghai.

\subsection{Motivation}

As the fast development of the Chinese high-speed trains, the reliability and safety of the high-speed trains are crit-

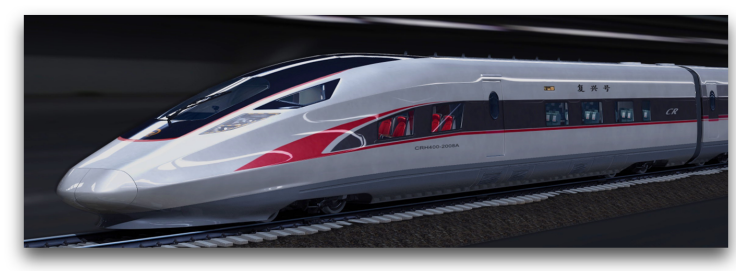

Figure 1. Fuxing Hao

ical for multiple-unit train (EMU) further development. However, there is no efficient fault detection and health monitoring methods which are really applicable in EMU trains due to the lack of failure data samples. And the high cost and difficulty of the experiment test of failure makes the health monitoring of EMU more difficult to achieve. In this paper, a modelica-based model is developed to generate fault samples for health monitoring.

\subsection{Overview of the paper}

As depicted in figure 2, the Chinese high-speed train consists of two parts: plant system containing traction system, braking system, auxiliary system and so on; and control system including network system, safety detection system. etc. In this paper, the braking system is used for demonstrating the sample identification method. First, we give a short background of the Chinese railway development and the motivation for the new health monitoring method proposed in this paper. Then the failure of the braking system of EMU was analyzed in the motivational example. Afterwards, the theroy of the sample identification method was explained in the technical background of fault diagnosis. Besides the theory of the proposed method, a demonstration of fault sample generation and health monitoring was also dicussed to verify the sample identification method, explicitly. In the presented study, a braking system with braking control unit (BCU), electromagnetic valve, reverse valve, double check valve and distribution valve were implemented with Modelica in Dymola. The health monitoring for the pressure and delay time of the reverse valve in the relay valve has been performed to illustrate the proposed approach. 


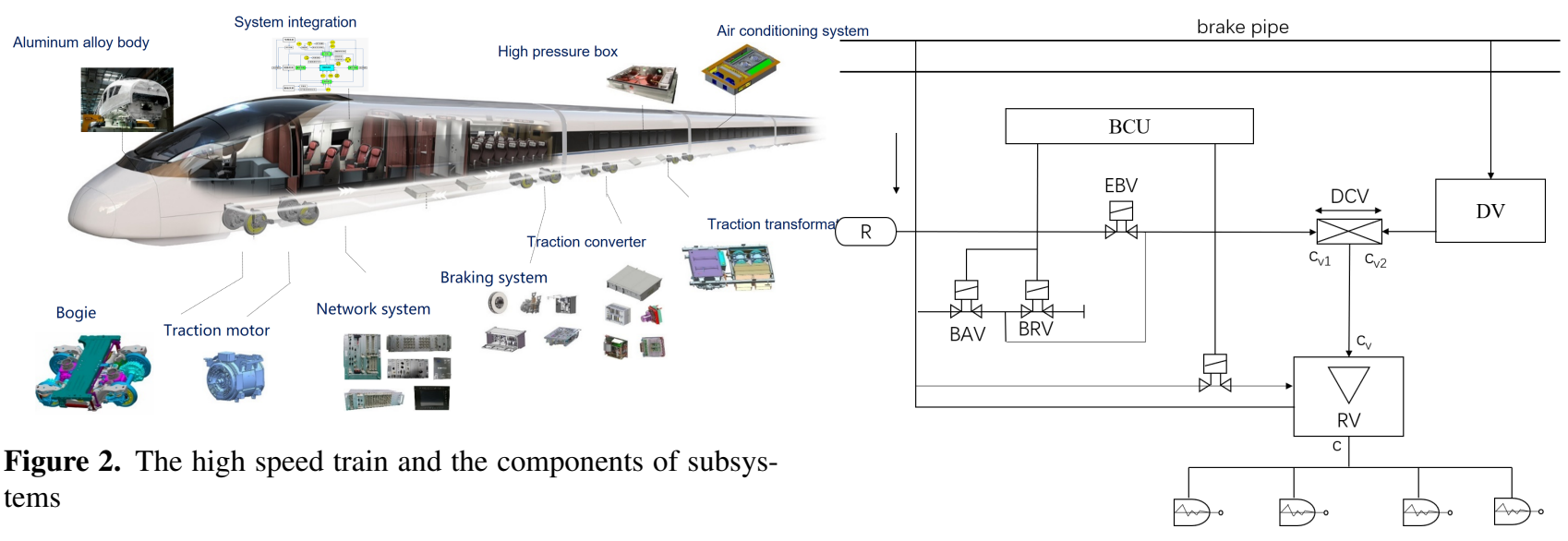

\section{Motivational Example}

\subsection{Introduction of the EMU braking system}

Braking is divided into two forms: dynamic braking and friction braking. Dynamic braking transforms the kinetic energy into electrical energy by changing the traction motor into a generator in braking. Disk braking is the most widely used in the friction braking (2). The EMU braking is achieved through the combination of electric braking and pneumatic braking, because the kinetic energy is too large for electric braking to consume in the limited time. The braking system can realize multiple functions such as service braking, emergency braking, urgency braking and so on. This paper takes focus on the urgency braking in which only pneumatic braking is applied to the train. The EMU braking system consists of three subsystems: braking control system, air supply system and braking devices. For the pneumatic braking, control system is the critical part that directly influence the braking performance of the train. As shown in figure 3, the braking control system consists of braking control unit (BCU), electromagnetic valve (BAV, BRV and EBV), reverse valve (RV), double check valve (DCV) and distribution valve (DV). The electromagnetic valve activates the electro-pneumatic braking and the distribution valve activates the pneumatic braking. The electromagnetic valve and distribution valve both produce the pre-controlled pressure $C_{v}$, the double check valve can ensure the transformation from $C_{v}$ to actual braking force. The braking cylinder pressure $C$ was adapted by reverse valve according to the $C_{v}$ value and accommodated the air volume of cylinder.

\subsection{Description of the failure to be analyzed}

In the emergency braking mode, a failure of changeover on high and low speed occurred occasionally, resulting in a low braking force during the braking, thereby prolonging the braking distance. Through the preliminary analysis of the fault performance and braking system principle, it is considered that relay valve of the braking control module causes the fault, and the reason is that the switching resistance of the relay valve is too high. In the braking control system, the relay valve (shown in figure 4)
Figure 3. Control system of braking system

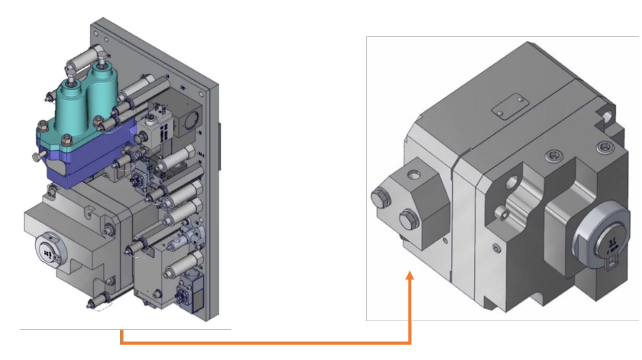

Figure 4. Relay valve

outputs the braking cylinder pressure complying with the requirements of the brake command by high-low speed changeover signal. After further analyzing the working principle of the relay valve, the cause of the fault is located in the reverse valve of the relay valve responsible for controlling the switching of the high/low speed braking force, as shown in figure 5. The reverse valve works as the following principle: when the train is applied brake at high running speed, compressed air pushes the changeover piston to right side to charge air into relay valve's pressure adjusting chamber, and relay valve outputs a lower braking pressure, which means lower braking deceleration of the train. While the running speed is decreased, compressed air in left side's chamber of changeover piston is cut off and vented, and the piston is pushed by return spring's force so that compressed air in pressure adjusting chamber of relay valve is vented, and control the relay valve to output a higher braking pressure, and of course, higher braking deceleration to the train. Failure

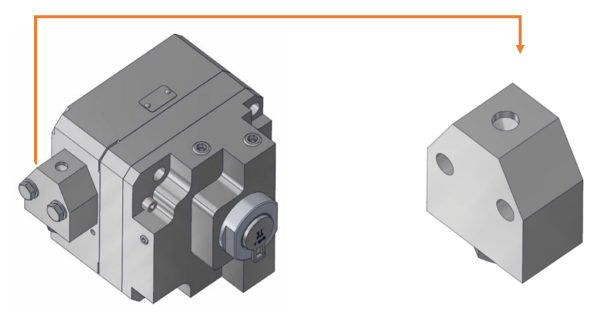

Figure 5. Reverse Valve 


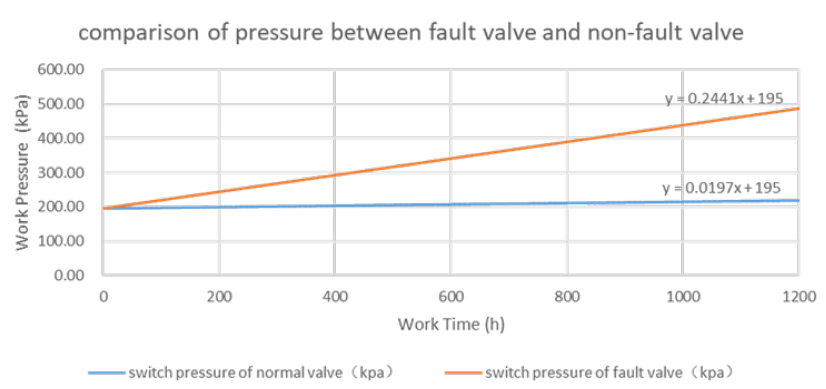

Figure 6. Pressure of fault valve and non-fault valve

phenomenon indicates that the piston can not be restorable by right side spring when the piston's left side pressure is vented. The reasons include: the excessive piston movement resistance; spring failure; small spring force. Because the failure is accidental, the piston can be finally restorable by right side spring in most cases, which indicates that this phenomenon is not caused by spring failure. So it should be caused by excessive resistance of valve stem. Finally, it was confirmed by experiments that the compatibility of the piston's rubber articles and lubricating grease was poor, resulting in excessive expansion of the outer dimensions, which further increased the friction force and verified the previous analysis. It can be seen from the experimental results that as the number of use time changes, the pressure required for switching gradually increases, and the increase amount in the fault valve is significantly higher than that of the normal valve, as shown in figure 6 .

\section{Technical background of fault diag- nosis}

\subsection{Traditional methods}

A fault is defined as an unpermitted deviation of at least one characteristic property or parameter of the system from the acceptable/usual/standard condition (3). In order to improve the reliability and safety of systems, fault diagnosis is introduced to monitor, locate and identify the faults. Traditional model-based method includes unknown-input observers approach (4), parity relation approach (5), stable factorization approach (6), Kalmanfilter-based residuals (7), parameter estimation method (8), distributed fault diagnosis filtering method (9), etc. The observer based method is widely used in fault diagnosis and health monitoring as shown in figure 7.

\subsection{Sample identification based approach}

This study addressed a new health monitoring method based on the sample identification. First step is to build the system models. After analyzing the reasons for the failures, a system model with no faults was built including the components in which failure occurred and other subsystems concerned in the fault diagnosis. Concerning

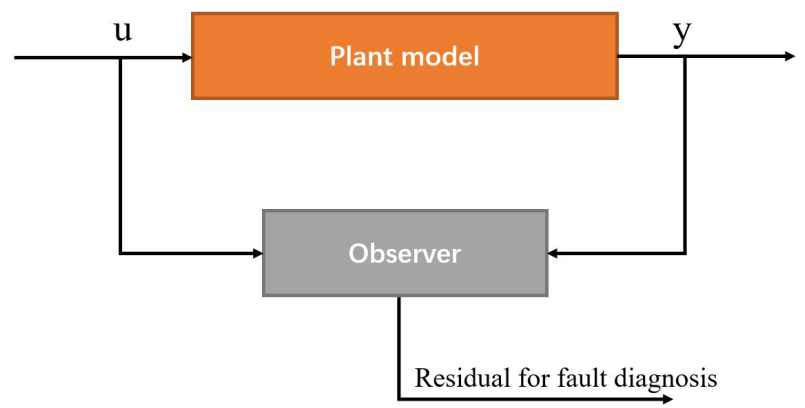

Figure 7. Schematic of model-based fault diagnosis

the high accuracy demand of the fault diagnosis and the complexity of the plant model, the modeling tool should be able to cover multiple physical domains and realize multi-level modeling with fast simulation and promised accuracy. Modelica is a technology modeling the dynamic behavior of technical systems consisting of components from, e.g., mechanical, electrical, thermal, hydraulic, pneumatic, fluid, control and other domains in a convenient way. Dymola is a tool based on Modelica with fast simulation and high accuracy which is used in this study. The model should be simplified as much as possible without decreasing the accuracy of fault diagnosis. And the healthy models should be validated for ensuring the accuracy of fault samples. At the same time, the corresponding fault models should be developed and be able to substitute the non-fault models in the healthy system models. Then, by inject the fault models into the healthy system models, the fault samples can be generated. As modelica can realize the multi-level modeling conveniently, it's very easy to change the healthy models by fault models. Next, distract the character value of the fault models which indicating the health degrees of the system concerned. In this study, the character is the delay time of braking pressure and the pressure of the reverse valve, the delay time defines the healthy degrees $(0 \sim 1)$ and pressure determines if the train is healthy (1) or failure (0). Finally, the fault samples were stored in the health monitoring server for computing the health degrees of the train based on the realtime detected data during the operation of the train. The algorithm for health monitoring is to compare the realtime data with the fault samples and determines the healthy degrees of the train or related subsystems.

The relationship of health degree with braking pressure $P$ and delay time $T$ can be generated by the considerable samples, as shown in equation 1.

$$
H=g(T)
$$

The braking signals and health degrees can be detected in real time, and analyzed to obtain the health state of the train. There are many algorithms for the health detectors, in which a simple one is utilized in this study: the delay time is the difference of braking operation time $T_{1}$ and signal output time $T_{2}$, as shown in equation 2 : 


$$
T=T_{2}-T_{1}
$$

The command is the braking boolean signal in the model

$$
T_{1}=\text { if command then time }
$$

The measured braking pressure is $P$, and $\tilde{P}$ was obtained after low pass filter, if the braking pressure was controlled to the desired pressure with no waves, the pressure should be above the threshold $a$ and the difference of the pressure should be below threshold $b$, as shown in equation 4 :

$$
T_{2}=\text { if } j \text { then time }
$$

Where $j$ is defined in equation 5:

$$
j=\text { if }(\tilde{P}>a \text { and } \operatorname{der}(\tilde{P})<b)
$$

and the relationship of braking pressure $P$ and delay time $T$ with health degrees $H$ is shown in equation 6:

$$
H=J(P, T) g(T)
$$

Where $J(P, T)$ is described in equation 7:

$$
J(P, T)=\text { if }(\text { command and } \tilde{P}>a)
$$

As the description above, the health monitoring was implemented and demonstrated in the following chapter.

\section{Case study}

\subsection{Modeling and validation of braking sys- tem}

The Chinese EMU is composed of 8 car units, divided into 2 traction units, each includes 2 driven cars and 2 trail cars, every driven car has a traction control unit (TCU) and every car unit has a braking control unit (BCU). BCU adopts the electrical-pneumatic braking controlled by TCU and BCU. The electrical braking is preferred and pneumatic braking is applied when the braking force is insufficient. BCU sends commands to air supply system according to the braking level set by driver, actual velocity of the train and pressure value detected by the braking system. A BCU model is shown in figure 8 . Technically, drivers can apply the braking without level set on the screen, but it's difficult to find appropriate operation position which is important to establish the reflection of driver. So braking is divided into service braking with 7 levels (B1 B7), emergency braking (EB) and urgent braking (UB) which applies mechanical braking only. In practice, the signals received by braking system are non-level continuous signals. The mechanical braking system is modeled based on the PneumaticLib developed by Modelon AB, as shown in figure 9, consisting of parking control unit, braking control unit, air supply and air spring control unit, pressure switches and braking executing unit. The braking execution unit of the driven car consists of four wheel discs

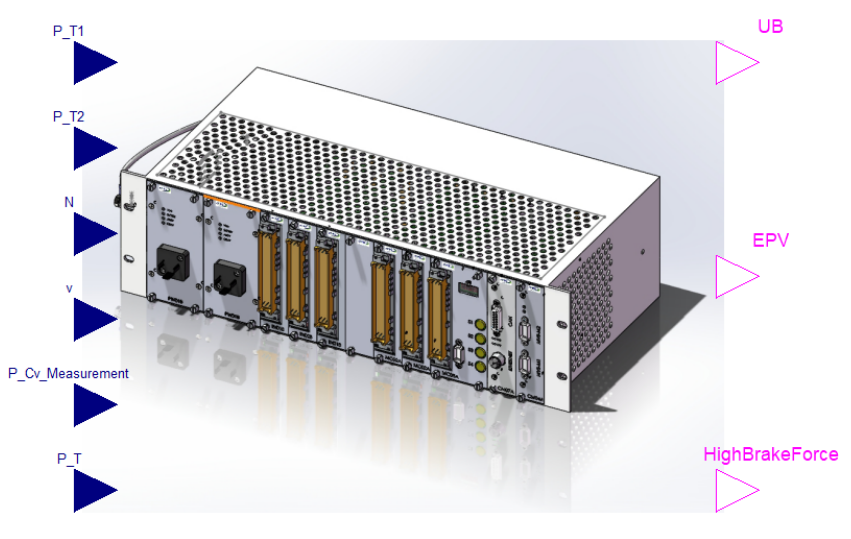

Figure 8. BCU model

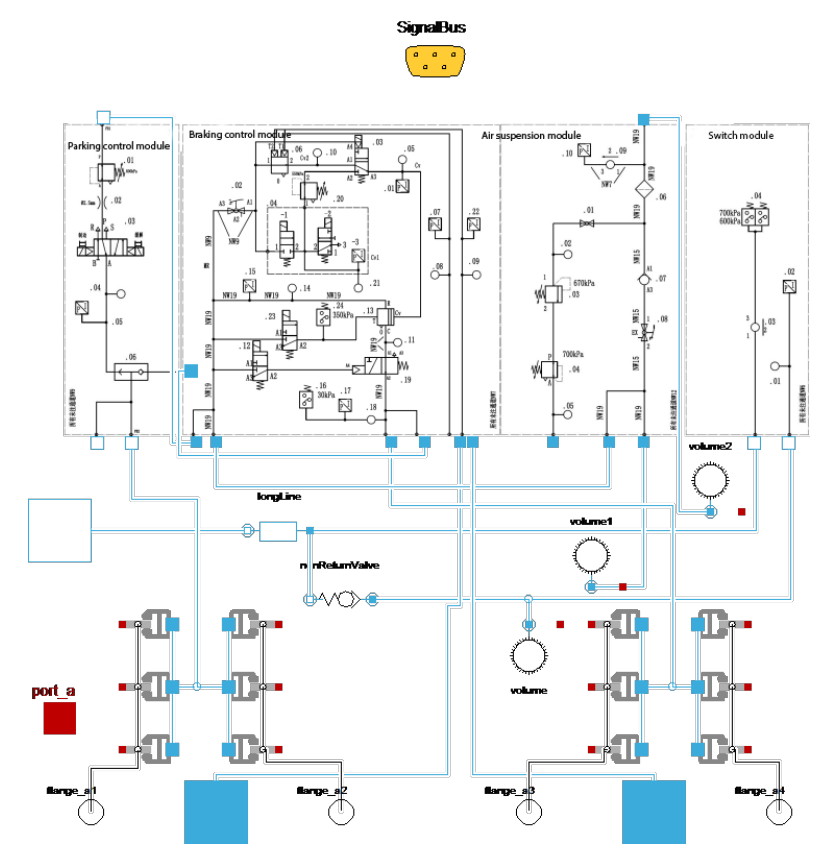

Figure 9. Braking system of the trail car TC01

per wheel set, and for the trail car, the braking execution unit is six hub discs. The main working unit is BCU. The braking system of the car TC01 is shown in figure 9. The BCU is as shown in figure 10, in which there are 3 special valves: EP valve used for adjusting and stabilizing the pressure quickly, empty and load valve used for controlling and adjusting the pressure according to the loads, relay valve used for switching between service braking and urgent braking. The models are described as follows: The details of EP valve model in figure 10 is shown in figure 11, consisting of 2 direction control valves (DCV_2_2). The pressure between the two valves is adapted by the switching operation of the direction control valve. The empty and load valve was depicted in figure 12, whose main function is to receive the air spring pressure signals of 2 bogies and output the braking control pressure that is linear to the load pressure value based on the leverage effect, so that the braking cylinder control pressure varies with the load in the braking mode. The mechanism of the 


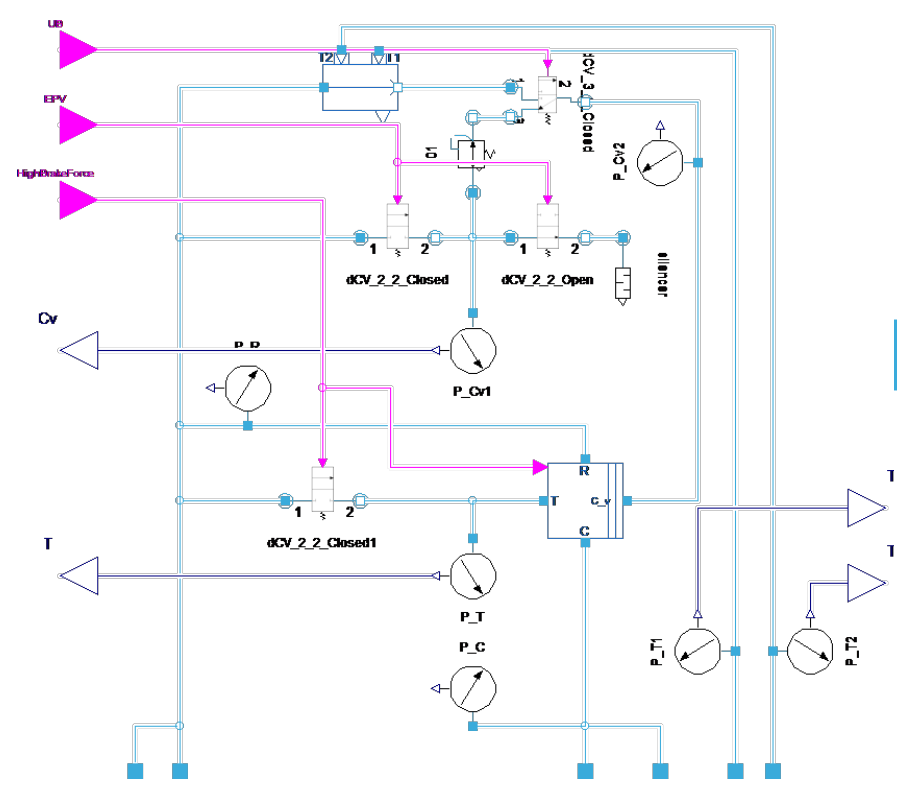

Figure 10. Braking control unit

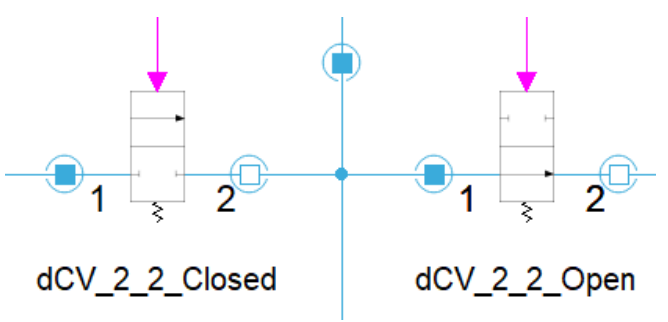

Figure 11. EP valves

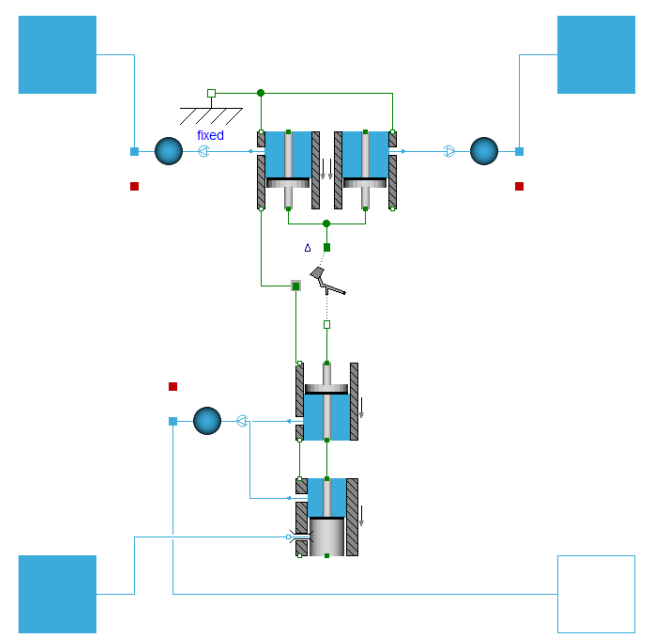

Figure 12. Empty and load valve model

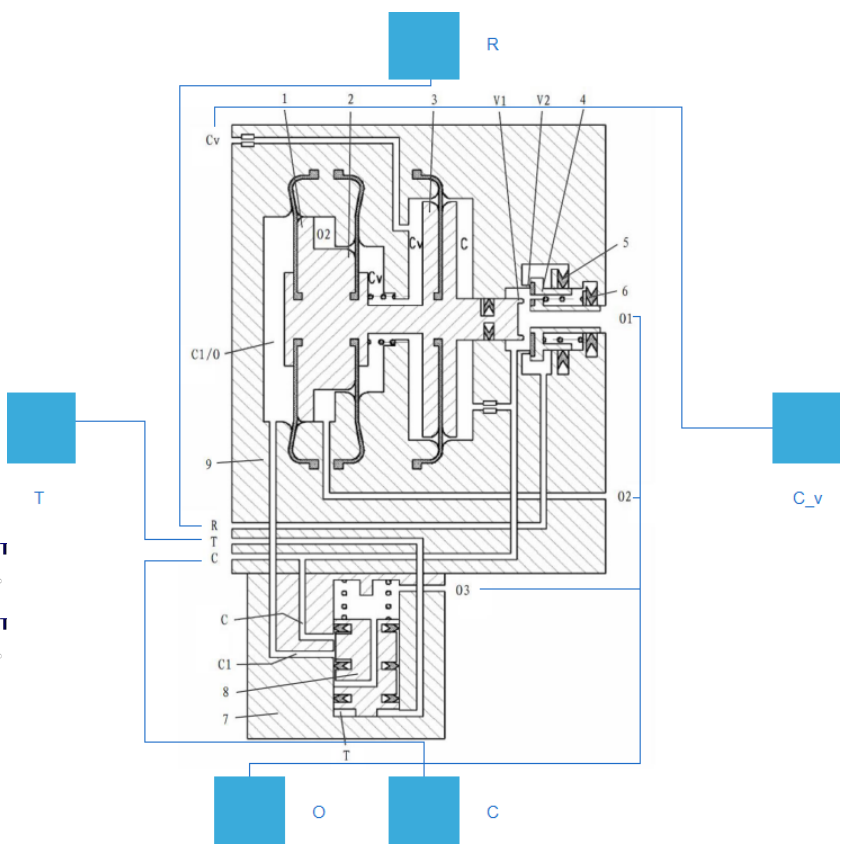

Figure 13. Mechanism of relay valve

relay valve is shown in figure 13 , whose main function is to increase the flow rate and ensure the output pressure $C$ varies with pressure $C_{v}$. When the train is running at high speed, reverse valve is closed, relay valve output low braking pressure; when the train is running at low speed, reverse valve is open and relay valve outputs high braking pressure. The reverse valve in normal operation is shown in figure 14. The model is validated by experiment data, as shown in figure 15. The input of BCU is the control signal from driver and actual speed of the train. Given that the driver operated in levels and the input signal of the BCU is continuous, the calculating results were revised and validated its consistency with test data. The formula of the validation is shown in equation 8 , where $T_{C}$ is the sample period, $X_{n}$ is revised value, $X_{\text {meas }}$ is test data and $X_{\text {simulation }}$ is calculating results. The validation results is shown in figure 16 indicating that the model is accurate enough for fault diagnosis and health monitoring.

$$
\frac{1}{T_{c}} \int_{t}^{t+T_{c}} \frac{\left|X_{\text {meas }}(u)-X_{\text {simulation }}(u)\right|}{\left|X_{\text {meas }}(u)\right|+0.01 X_{n}} d u \leq 0.05 \forall t
$$

\subsection{Fault injection and failure sample genera- tion}

The fault caused by the reverse valve of the relay valve is performed as time delay of the switch between high and low pressure. Specifically, the reason is that the coefficient of friction is too large for the moving valve. The fault models of reverse valve are injected into the healthy model through changing the coefficient of friction for the fault diagnosis modeling. The fault models of reverse valve is shown in figure 17. The same control signal is applied to the models with fault injection, and with different coefficient of friction, different braking pressure is obtained, the 


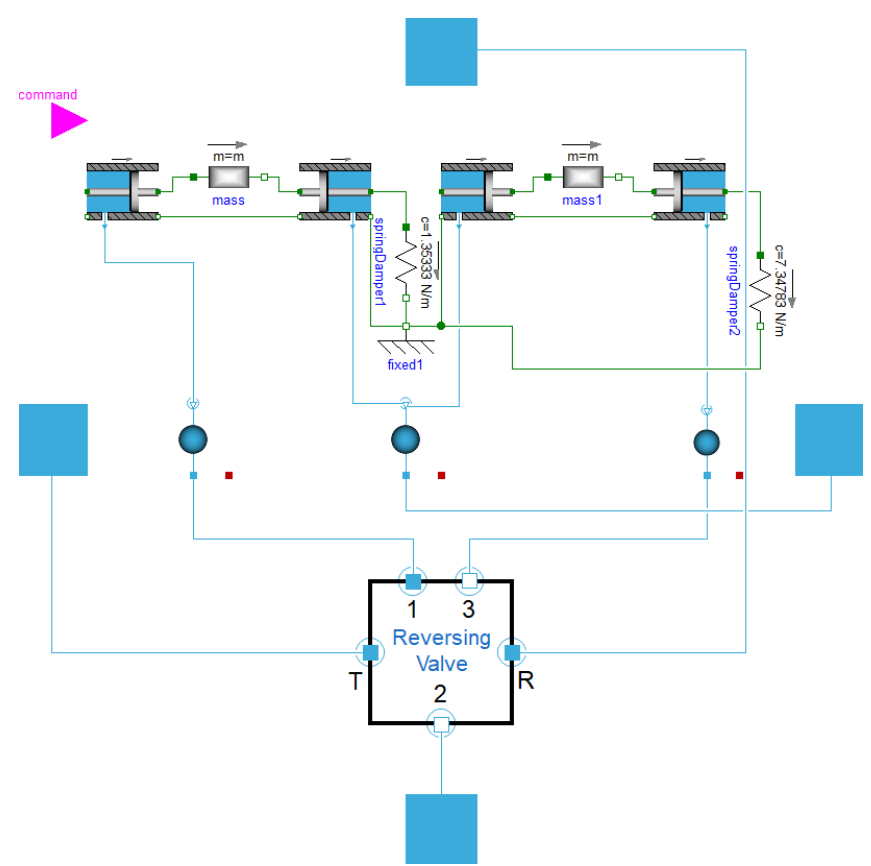

Figure 14. Relay valve model in normal operation

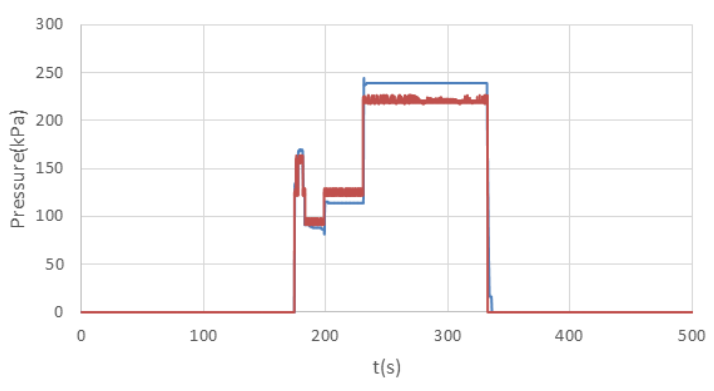

- Simulation Result of Pressure $\quad$ Measured Pressure

Figure 15. Validation results of the braking pressure

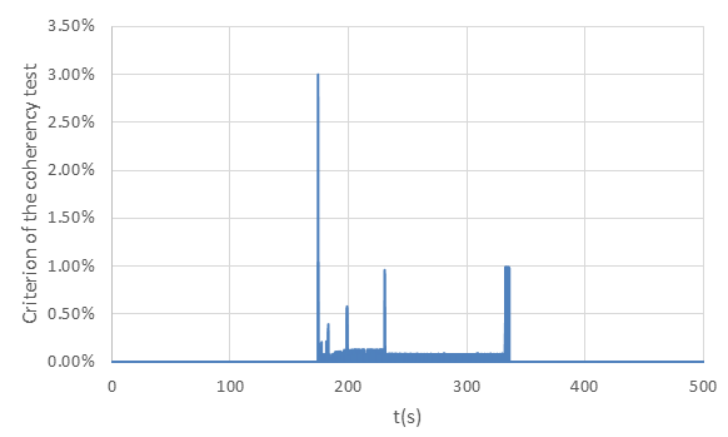

Figure 16. Relative error of the coherency validation

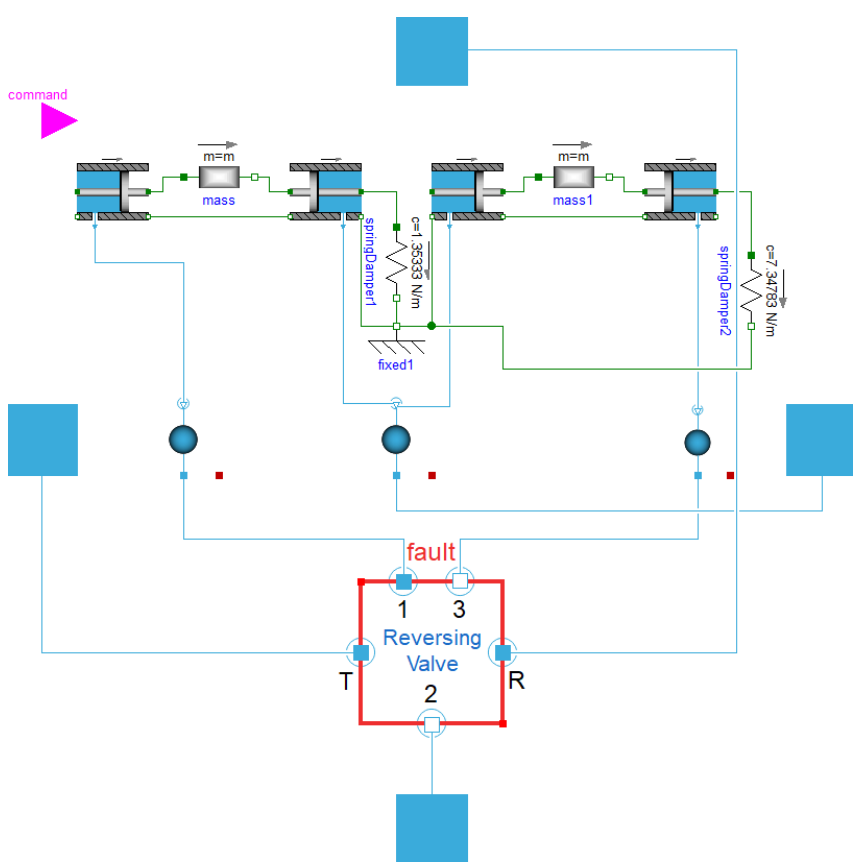

Figure 17. Relay valve with faults

time of the reverse valve to complete the switching operation is delayed, or even can't switch normally. Specifically manifested in three forms as follows: smooth switching between high and low pressure, slow switching between high and low pressure, failed switching between high and low pressure. When there are switching delays or failures that reverse valve can not be switched properly, the braking distance of the failed train is longer than that of the fault-free train, as shown in figure 18.

\subsection{Application of sample identification based health monitoring}

The faults in the train have bad impacts on the performance, life cycle and stability of braking system. The unstable performance and component broken manifest immediately, and performance degradation is difficult to notice which may cause severe consequences once the failure occurred. It's critical to monitor the system states online in real time ensuring the normal operation in healthy condition. The fault distribution is assumed as normal distribution. The healthy degree is 1 when the coefficient of friction is 0 in which the probability is biggest. And the health degree and probability decrease with coefficient of friction increases, as shown in figure 19. However, considerable samples are essential in the process of the monitoring online system development, which are generated by injecting different coefficient of friction into models with the same pneumatic switch signals, as shown in figure 19, 20. The figure 21 depicts different braking cylinder pressure and abstract the delay time of signals $T$ and braking pressure $P$ to analyze and decide the health degree. The simulation results of the test case by applying the proposed sample identification based approach is shown in figure 22. More sophiscated control algorithms for fault diagnosis can be 


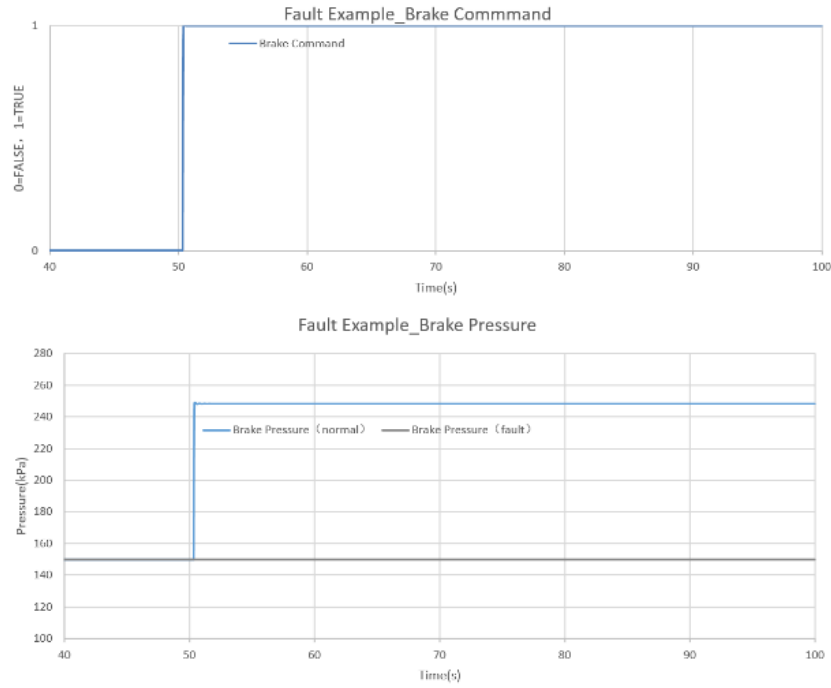

Figure 18. Simulated results when the high and low pressure failed to switch

developed based on the considerable samples generated by the models, and applied in the health monitoring system development.

\section{Conclusion and future work}

In this paper, a sample identification method for the fault diagnosis and health monitoring is introduced. The models of braking system in a chinese high speed train with high accuracy and the corresponding fault models have been implemented. The faults were injected into the healthy models to generate a large amount of fault samples. The new health monitoring method has been demonstrated by a predefined failure in the braking system.

\section{Acknowledgment}

The authors gratefully acknowledge ZEMT for their help of providing the braking system test data. SIMTEK also cooperated with ZEMT on the modeling of other EMU subsystems such as traction system and control system and integrated all the key subsystems as a train for power consumption estimation and other studies.

\section{References}

[1] K. Barrow, "Ten years, 27,000km: China celebrates a decade of high-speed," International Railway Journal. August 2, 2018.

[2] WS Guan, JJ Wang, HS Wang, "The Braking Mode Simulation and Analysis for High-speed Railway", 4th IEEE International Symposium on Microwave, Antenna, Propagation and EMC Technologies for Wireless Communications, China: Beijing, 2011, pp.683-686.

[3] Z WG, C Cecati, et al, "A Survey of Fault Diagnosis and Fault-Tolerant Techniques-Part I: Fault
Diagnosis With Model-Based and Signal-Based Approaches", IEEE transactions on industrial electronics, vol.62, 2015, pp. 3757-3767.

[4] J. Chen and H. Zhang, "Robust fault detection of faulty actuators via unknown input observers,", vol.22, Int. J. Syst. Sci., 1991, pp.1829-1839.

[5] E. Chow and A. Willsky, "Analytical redundancy and the design of robust detection systems", IEEE Trans. Autom. Control, vol.29,1984, pp.603-614.

[6] N. Viswanadham, J. Taylor, and E. Luce, "A frequency-domain approach to failure detection and isolation with application to GE-21 turbine engine control systems", Control, Theory Adv. Technol., vol.3, 1987, pp. 45-72.

[7] S. Helm, M. Kozek, and S. Jakubek, "Combustion torque estimation and misfire detection for calibration of combustion engines by parametric Kalman filtering", IEEE Trans. Ind. Electron., vol.29, 2012, pp. 4326-4337.

[8] A. Akhenak, E. Duviella, L. Bakoa, and S. Lecoeuchea, "Online fault diagnosis using recursive subspace identification: Application to a damgallery open channel system", Control Eng. Pract., vol.21, 2013, pp. 797-806.

[9] C. Keliris, M. Polycarpou, and T. Parisini, "A distributed fault detection filtering approach for a class of interconnected continuous-time nonlinear systems", IEEE Trans. Autom. Control, vol.58, 2013, pp. 20322047. 


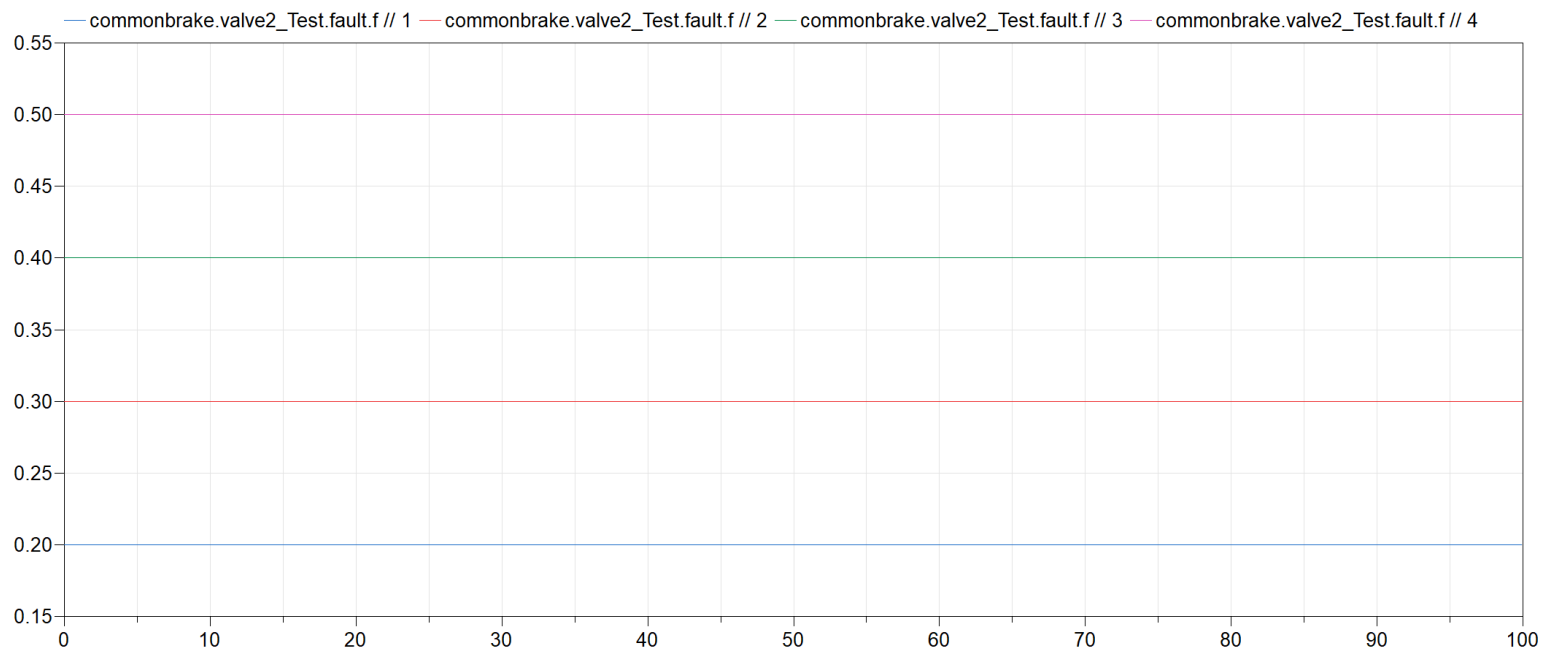

Figure 19. Coefficient of friction when injecting faults

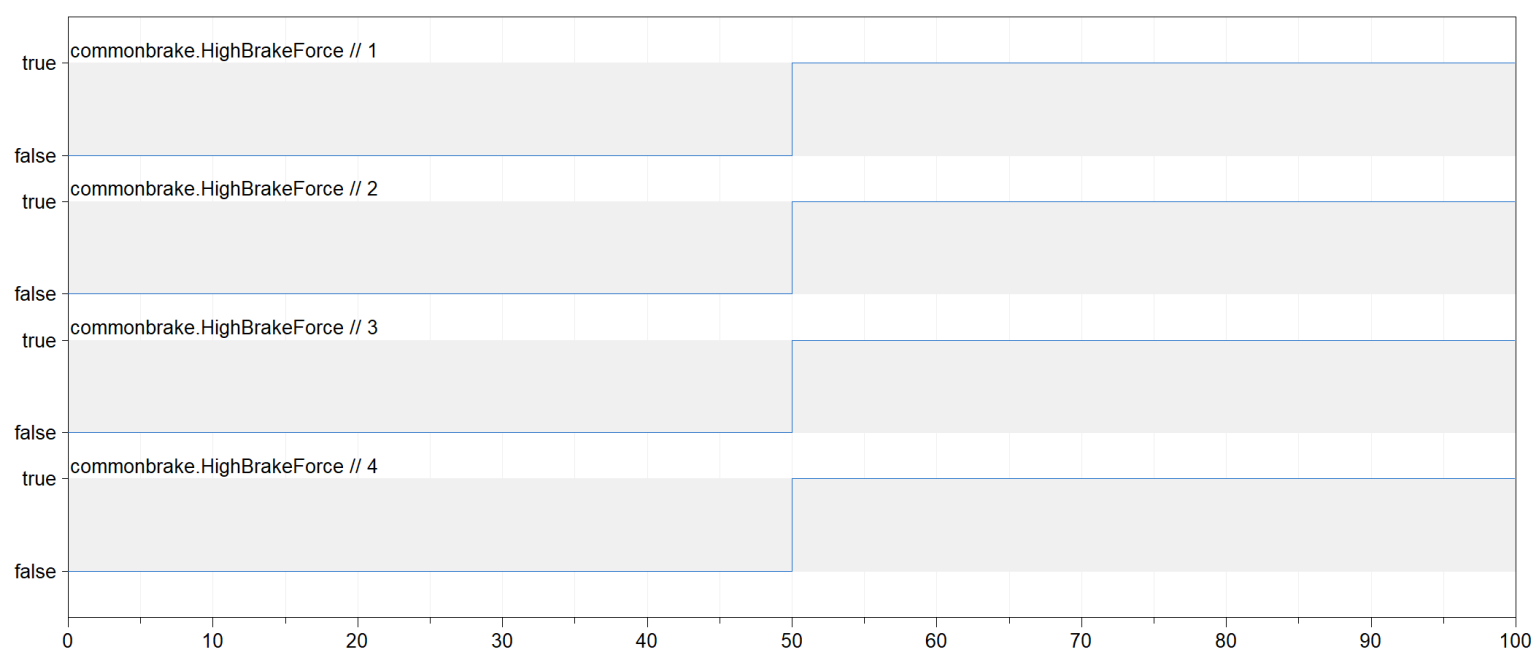

Figure 20. Pneumatic switch signals

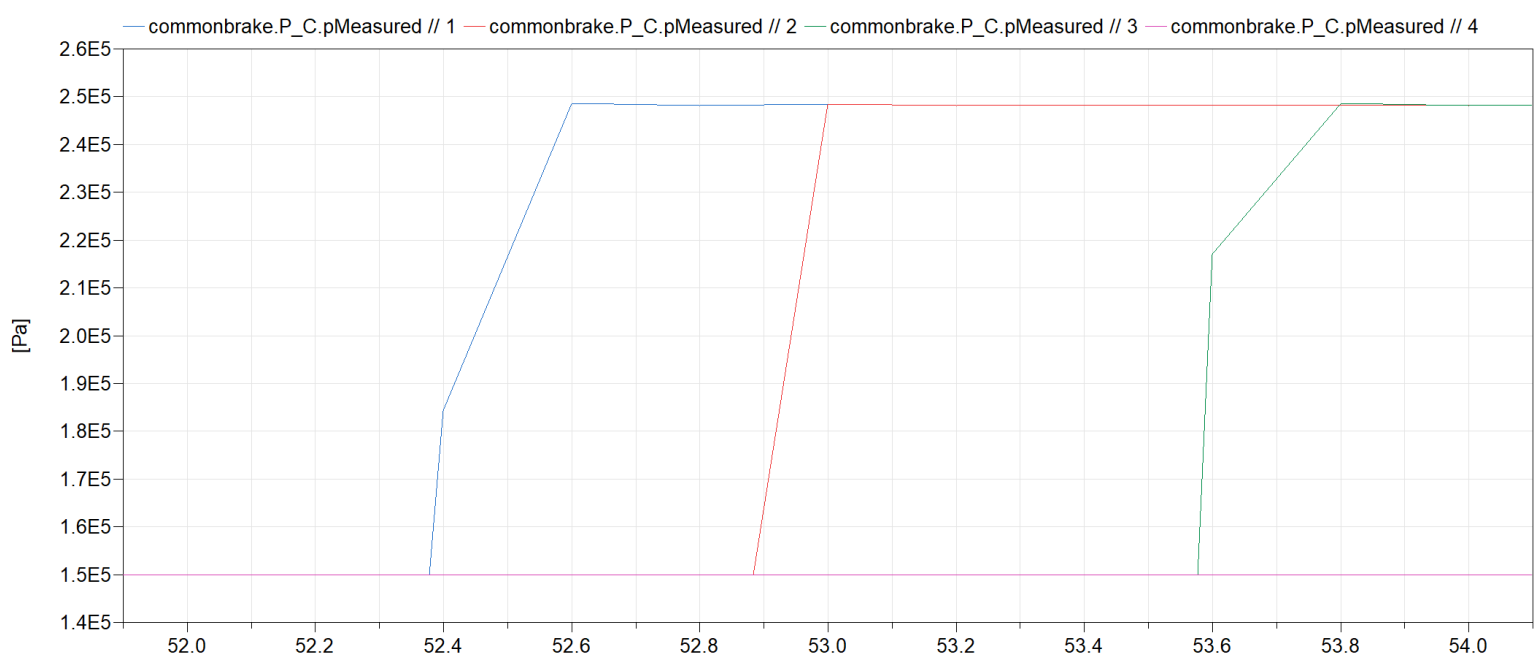

Figure 21. Braking pressrue relative to different coefficients of friction 
Model based Health Data

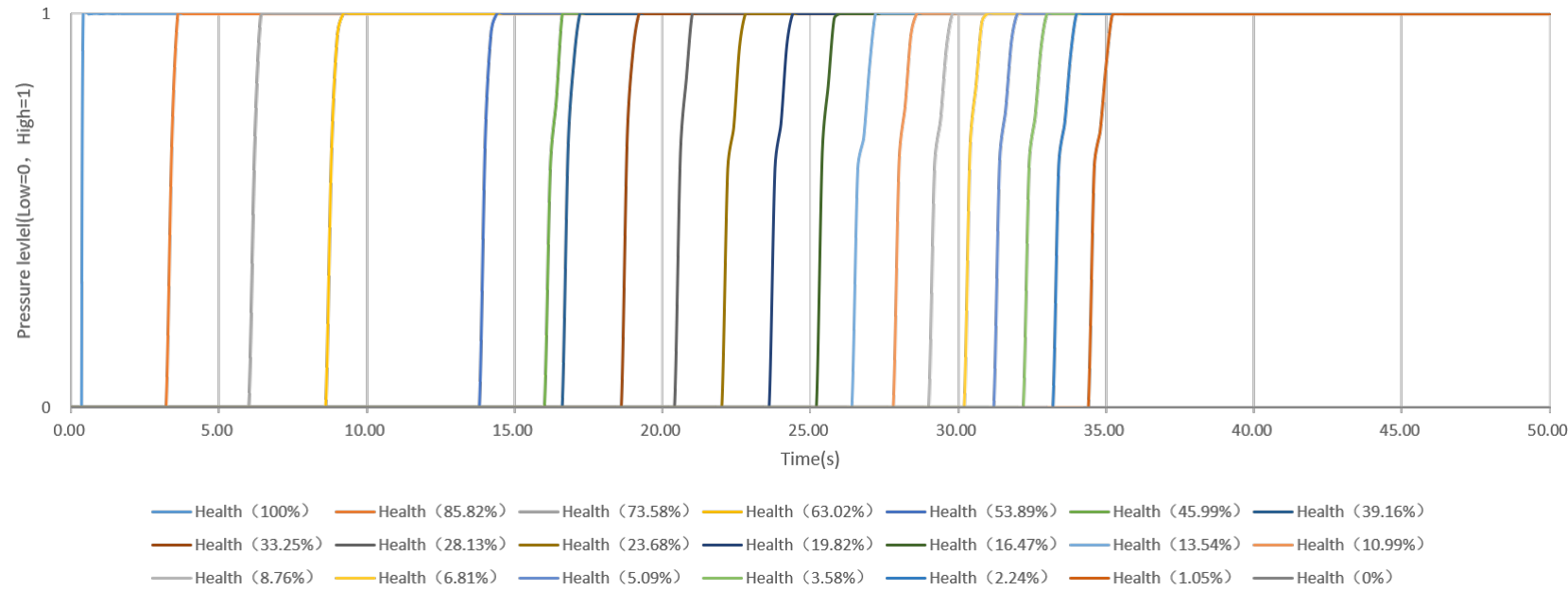

Figure 22. Different health degrees decided by multi samples generated by fault injection 